\title{
The fear and anger model of mood, behavior and personality Diogo Rizzato Lara
}

\author{
Address: Catholic University of Rio Grande do Sul, Porto Alegre, Brazil \\ from International Society on Brain and Behaviour: 3rd International Congress on Brain and Behaviour \\ Thessaloniki, Greece. 28 November - 2 December 2007 \\ Published: 17 April 2008 \\ Annals of General Psychiatry 2008, 7(Suppl I):S36 doi:10.1186/1744-859X-7-SI-S36
}

This abstract is available from: http://www.annals-general-psychiatry.com/content/7/SI/S36

(C) 2008 Lara; licensee BioMed Central Ltd.

Current formal psychiatric approaches to nosology are plagued by an unwieldy degree of heterogeneity with insufficient appreciation of the commonalities of emotional, personality, behavioral, and addictive disorders. This challenge is addressed by building a spectrum model that integrates the advantages of Cloninger's and Akiskal's approaches to personality and temperament while avoiding some of their limitations. We specifically propose that "fear" and "anger" traits - used in a broader connotation than in the conventional literature, including drive and desire - provide an optimum basis for understanding how the spectra of anxiety, depressive, bipolar, ADHD, alcohol, substance use and other impulse-control, as well as cluster $\mathrm{B}$ and $\mathrm{C}$ personality disorders arise and relate to one another. By erecting a bidimensional approach, the paradox that apparently polar conditions (e.g. depression and mania, compulsivity and impulsivity, internalizing and externalizing disorders) can coexist without cancelling one another can be resolved. The combination of excessive or deficient fear and anger traits produces 4 main quadrants corresponding to the main temperament types of hyperthymic, depressive, cyclothymic and labile individuals, which roughly correspond to bipolar I, unipolar depression, bipolar II and ADHD, respectively. Other affective temperaments resulting from excess or deficiency of only fear or anger include irritable, anxious, apathetic and disinhibited/hyperactive. Our model does not consider schizophrenia and autism. We propose that "healthy" or euthymic individuals would have average or moderate fear and anger traits ("anger" most expressed as drive rather than anger itself). We further propose that family history, course and comorbidity patterns can also be understood based on fear and anger traits. This model has implications for clinical diagnosis of the common psychiatric disorders, and for subtyping depression and anxiety as well as cognitive and behavioral styles. Pharmacological treatments with antidepressants and anxiolytics can be considered as essentially restraint on fear, whereas lithium would attenuate fear deficiency. Dopaminergic antidepressants and psychostimulants are anger/drive enhancers and antipsychotics and mood stabilizers, such as divalproate and carbamazepine, may share antianger effects. Drugs effective for manic and depressive phases probably have both antianger and antifear effects. Nonpharmacological treatments would also mediate their effects by acting on fear and anger/drive. The preliminary data testing this model will be shown and discussed. 